\title{
El desarrollo del conocimiento humano sobre el tiempo
}

\author{
M. ${ }^{a}$ BelÉn BuENO \\ Universidad de Salamanca
}

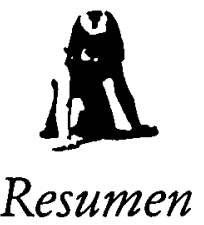

\begin{abstract}
La investigación que existe en tomo al desarrollo del conocimiento bumano sobre el tiempo muestra que en los primeros años de vida los niños adquieren información importante sobre la temporalidad que les posibilita una adaptación adecuada a su ambiente y el desarrollo de conductas afectivas, perceptivas, cognitivas, lingüisticas y sociales fundamentales. Además, muestra que cuando se consigue el dominio de las herramientas cognitivas y lingǘsticas en torno a los 6-8 años de edad, no se adquiere un único concepto sobre el tiempo, sino que se conocen diversos aspectos del tiempo dificiles de ser aunados en un concepto único, todos los cuales son necesarios para el funcionamiento adecuado en el mundo de compleja estructura en que nos encontramos. Tras las últimas modificaciones ocurridas en la adolescencia, sobre todo en lo que se refiere a la perspectiva temporal, el razonamiento sobre el tiempo adquiere las características de la cognición temporal adulta.
\end{abstract}

Palabras clave: Desarrollo del conocimiento bumano sobre el tiempo, Habilidades temporales, Concepto de tiempo, Cognición temporal, Estructura temporal.

\section{The development of human's knowledge of time}

\section{Abstract}

Current research on the development of human's knowledge about time shows that during the first years of life children acquire important information about temporality that facilitates, on the one band, andequate adaptation to their environment, and, on the other, the development of fundamental affecti. ve, perceptual, cognitive, linguistic, and social kinds of bebaviour. Furthermore, findings show that when mastery of cognitive and linguistic tools are achieved, at about 6-8 years of age, it is not a single concept of time that is acquired but rather diverse aspects of time that are difficult to encompass within a single and unique concept. All of these are necessary for proper functioning in a world so structurally complicated as the one in which we live. After the modifications which take place in adolescence, especially from a temporal perspective, time reasoning acquires characteristics of adult temporal cognition.

Keywords: Development of human's knowledge of time, Temporal ability, Time concept, Temporal cognition, Temporal structure.

Agradecimientos: Este trabajo ha sido realizado gracias a una beca predoctoral concedida por la Universidad de Salamanca.

Correspondencia con autor-es: Departamento de Psicología. Avda. de la Merced, 109-131. 37005 - Salamanca.

Original recibido: Junio, 1991. Revisión recibida: Marzo, 1992. Aceptado: Junio, 1992. 


\section{INTRODUCCION}

La investigación sobre el desarrollo cognitivo tiene una primera etapa, a menudo implícita, que supone la delimitación de un dominio conceptual. Esa tarea se lleva a cabo desde el punto de vista del adulto y los límites que se establecen reflejan la epistemología del investigador, no la del niño. El tiempo psicológico, núcleo temático de este trabajo, es un concepto que para los adultos tiene un dominio amplio, aunque con unos límites hasta cierto punto discutibles puesto que no existe consenso sobre cómo y cuándo se desarrollan las diversas nociones, conceptos o ideas sobre el tiempo.

Hasta la actualidad, la investigación psicológica sobre el tiempo ha crecido en direcciones diferentes y no relacionadas. Mientras que el punto de partida para muchos de los estudios evolutivos del tiempo en niños fue el trabajo de Piaget (1946), modelos de almacenamiento y de atención, tales como el de Ornstein (1969), el de Hicks, Miller y Kinsbourne (1976) ó el de Block (1978) han servido de base para los estudios cognitivos sobre el tiempo en los adultos (Bueno, 1990). En relación con la cognición temporal nuestro interés radica en que el estudio psicológico del tiempo no se sustraiga a un enfoque pluralista en el que se aborde el desarrollo desde planteamientos amplios e integradores.

Resulta difícil construir o inferir una perspectiva integrada por cuanto el campo se ha organizado en grupos de intereses específicos con poca integración teórica. La investigación está representada todavía por estudios más o menos intensivos de problemas particulares en períodos concretos del curso vital, especialmente en la edad adulta. Además, dado que durante mucho tiempo se ha pensado que el conocimiento humano sobre la temporalidad exigía el dominio de las nociones simbólicas y lingüísticas debido a su nivel de abstracción, apenas si se ha investigado el desarrollo de las habilidades temporales de los niños muy pequeños.

Puesto que para describir y explicar la conducta de los seres humanos es fundamental conocer la forma en que ésta se desarrolla y cambia, nuestra intención ha sido destacar la adquisición gradual del conocimiento humano sobre el tiempo hasta la consecución de su dominio completo, así como mostrar el abanico de conocimientos que sobre la temporalidad esgrimen los niños más mayores.

Como se verá, existe una nítida diferencia en la naturaleza de las cuestiones planteadas y en la metododogía empleada por los investigadores en relación con la temporalidad puesto que, tal y como defienden Fraisse $(1957 / 1963,1982)$ y Montangero (1984), hay que distinguir entre distintos modos de adaptación al tiempo. Cada uno de esos niveles de adaptación contribuiría a la adquisición del nivel siguiente en la progresión del ser humano para conseguir, en última instancia, el dominio de la noción de tiempo, logro intelectual que supone una gran abstracción.

Por un lado estaría el condicionamiento al tiempo, en la medida en que, como el resto de los animales, somos sensibles a los ritmos de origen tanto endógeno como exógeno y nos condicionamos a los intervalos entre señales. Tanto unos como otros nos proporcionan información útil para orientarnos temporalmente a un nivel cognitivo y práctico. Por otro lado estaría la percepción del tiempo, que se refiere a la percepción de duraciones breves y mediante la cual podemos percibir y adaptarnos a cambios rápidos. En tercer lugar está la representación del tiempo que presenta dos niveles, uno que permanece al nivel preoperacional 
y que involucra la representación de cambios pasados y actuales, y otro que permite construir una representación exacta de esos cambios y anticipar y dominar los futuros (Fraisse, 1982).

Los investigadores de la primera infancia han estudiado los aspectos más elementales de la adaptación del ser humano al tiempo, aspectos considerados como los precursores de las habilidades temporales puestas de manifiesto por los niños más mayores. El condicionamiento a la duración y la percepción del tiempo figuran entre los intereses prioritarios de quienes se han interesado por el «conocimiento temporal» de los bebés. En contraposición, los investigadores interesados por el conocimiento temporal que poseen los niños más mayores se han centrado principalmente en habilidades de razonamiento general aplicadas al tiempo, dedicando una atención prioritaria a la adquisición de las propiedades ordinales, métricas y representacionales del tiempo en la medida en que los niños de 6-8 años poseen las capacidades lingüísticas y cognitivas necesarias para su dominio. Finalmente, en la adolescencia la investigación temporal se ha centrado en la consolidación de las habilidades hasta ese momento en desarrollo y en las peculiaridades de la perspectiva temporal humana en esa etapa.

Como es lógico, los contenidos teóricos sobre los que se investiga y la competencia de los niños de diferentes edades, condicionan la metodología empleada. Mientras que en la primera infancia, dadas las peculiaridades de esta etapa evolutiva, se investigan los ritmos circadianos o el grado de condicionamiento del bebé al tiempo mediante técnicas bioconductuales, en la segunda infancia se emplean métodos que exigen una participación más activa del niño tanto si se pregunta por la duración de los sucesos como si los problemas son de orden temporal. En este sentido, se han utilizado métodos de dificultad variada, desde requerir de los niños juicios relativos y explicaciones sobre el comportamiento de animales variando las dimensiones de posición, distancia y velocidad (problemas al estilo piagetiano), hasta simplificar las tareas solicitando sólo estimaciones directas de las dimensiones involucradas o variando de modo sistemático los parámetros de tarea utilizados, e incluso utilizando el entrenamiento y analizando la respuesta diferencial a él dada. Durante la adolescencia, generalmente se han utilizado entrevistas en las que se pregunta por cuestiones sobre la orientación temporal personal o sobre el resultado más probable de una situación inventada. Las medidas que se han utilizado incluyen escalas de categorías y análisis de técnicas proyectivas que incluyen representaciones lineales y circulares del tiempo.

Los trabajos que se revisan en este informe constituyen una clara ilustración de los diversos aspectos de la temporalidad que los seres humanos dominan a lo largo de su desarrollo, así como de la variedad de planteamientos teóricos y metodológicos que se manejan en este dominio epistemológico. A pesar de la complejidad del conocimiento temporal, la investigación sobre los distintos aspectos de su desarrollo puede desempeñar un papel importante en nuestra comprensión del desarrollo cognitivo. De cualquier modo, este artículo no pretende agotar el tema; lo que se intenta es sentar las bases evolutivas de los conocimientos temporales que diariamente manifestamos los adultos.

\section{LOS PRIMEROS AÑOS}

En claro contraste con el amplio cuerpo de conocimientos existente sobre el desarrollo de los conceptos de tiempo en los niños más mayores - no obstan- 
te la considerable controversia sobre la naturaleza específica de dicho proceso evolutivo-, existe un cierto vacío informativo sobre los precursores de esas habilidades en la primera infancia. Dicha falta de información resulta sorprendente si tenemos en cuenta que ya Piaget en 1954 consideró que el tiempo era junto con el espacio, el objeto y la causalidad una categoría fundamental de la experiencia mental de los niños. Además, la regularidad temporal o secuenciación de los sucesos en el mundo infantil arroja información importante para su funcionamiento adaptativo y su capacidad para funcionar en un dominio temporal es crítica para el desarrollo de conductas afectivas, lingüísticas, cognitivas, perceptivas y sociales (Lewkowicz, 1989).

A pesar de que es bien sabido que el estudio del desarrollo ontogenético arroja información importante sobre la organización y la función de la conducta, el acercamiento ontogenético ha estado muy olvidado en lo relativo a los ritmos bioconductuales, y, específicamente, a los ritmos circadianos (Davis, 1981; Estaun-Ferrer, 1985). Sin embargo, sabemos que el niño está sometido a los ritmos biológicos desde su nacimiento, siendo claros ejemplos de dichos ritmos los períodos sucesivos de vigilia y de sueño, así como los movimientos rítmicos y estereotipados que realizan los niños con pocos meses de edad (Thelen, 1979). En este sentido, Thelen encontró que la edad en la que los movimientos estereotipados ocurren con mayor frecuencia está entre los 6 y los 7 meses. Esta autora rechaza la argumentación piagetiana de que los niños se involucran en dichos movimientos - reacciones circulares secundarias en términos piagetianos - a causa de su conocimiento de la causalidad o de las consecuencias secuenciales de sus propias acciones. Thelen propone que los estereotipos son el producto de la generación de patrones espaciales y temporales intrínsecos en el sistema nervioso, lo cual no sería impedimento para que tales conductas tuviesen funciones instrumentales, entre las que figurarían la de ser precursores del desarrollo de la conducta habilidosa (Thelen, 1981), la de proporcionar control contingente sobre los cuidadores ó las de servir como vehículo de comunicación o para medir la duración de los sucesos (Pouthas, 1985).

Por otra parte, los factores temporales desempeñan un papel crítico en las interacciones sociales que los niños pequeños mantienen con las personas que los cuidan, tanto en aquellas que involucran actos comunicativos como en las que conllevan actos no comunicativos. En este tipo de estudios se ha encontrado que con unos pocos meses de edad, el niño se forma expectativas temporales en los intercambios comunicativos con su cuidador gracias a que la duración de las frases que éste emplea para comunicarse con el niño varía poco Jasnow y Feldstein, 1986; Stern, Beebe, Jaffe y Bennett, 1977), variaciones que, por otro lado, sirven para mantener la atención del niño (Stern y Gibbon, 1979).

De modo similar, algunos autores (Condon y Sander, 1974a, 1974b; Dowd y Tronik, 1986) han examinado la posibilidad de que los movimientos de diferentes partes del cuerpo de los niños muy pequeños estén sincronizados con el discurso de los adultos. Aunque se han obtenido algunos resultados conflictivos, parece ser que los niños de entre 3 y 5 meses de edad exhiben ritmos en sincronía con los manifestados por sus madres y que existe un aumento de las conductas sincrónicas de los niños nacidos tras los nueve meses de gestación en relación con las de los niños prematuros (Lester, Hoffman y Brazelton, 1985). Lester y sus colaboradores especulan sobre el origen de los ritmos observados mediante la propuesta de que tales ritmos proceden de los mecanismos de control homeostático autonómico que mediatizan el arousal del Sistema Nervioso 
Central y que mecanismos de cronometraje u osciladores neurales intrínsecos pueden controlar los ritmos conductuales que se observan.

También se ha encontrado que el condicionamiento a una duración periódica en niños recién nacidos es posible, resultado que viene avalado tanto por estudios pioneros como el de Marquis (1941), cuanto por estudios más recientes (Brackbill y Fitzgerald, 1972; Brackbill, Fitzgerald y Lintz, 1967; Brackbill, Lintz y Fitzgerald, 1968; Fitzgerald, Lintz, Brackbill y Adams, 1967), aunque parece ser que su éxito depende de factores tales como la modalidad del estímulo condicionado, la complejidad de la tarea, el sistema de respuesta utilizado, el sexo (Stamps, 1977; Stamps y Porges, 1975; Turco y Stamps, 1980) y la edad del niño, lo que dificulta el logro de conclusiones firmes sobre las capacidades temporales de los niños muy pequeños basándonos en este tipo de estudios.

Además del aprendizaje de que diversas señales neutrales predicen la ocurrencia de un estímulo incondicionado, hay muchas situaciones en el mundo infantil que presentan consecuencias cuando el niño pequeño se involucra en alguna actividad. Para que el niño pequeño aprecie la relación causal entre su acción y sus consecuencias, debe ser capaz de comprender que a menudo existe un retraso temporal aceptable entre su acción y sus consecuencias. En este sentido, se ha encontrado que un retraso de tan sólo 3 segundos en la administración del refuerzo que sigue a la ejecución de una respuesta evita la adquisición de una nueva respuesta (Millar y Watson, 1979; Ramey y Ourth, 1971). Puesto que tan severa limitación en la adquisición infantil de nuevas respuestas tendría efectos desastrosos sobre el desarrollo de la competencia conductual, es muy probable que existan factores diferentes a los temporales que ayuden al niño en la adquisición de nuevas respuestas. Watson $(1979,1984)$ ha propuesto una teoría que intenta explicar la capacidad infantil para analizar su eficiencia causal personal haciendo referencia no sólo a la información temporal, sino también a las semejanzas en la estructura sensorial de respuestas y estímulos. De cualquier modo, se ha visto que los niños de tan sólo 3 años de edad poseen los principios subyacentes al razonamiento causal. De aquí se deriva el hecho de que el desarrollo de la comprensión causal es más un proceso de aprender dónde, cuándo y cómo aplicar las reglas de razonamiento que uno de descifrar qué reglas podrían ser (Bullock, Gelman y Baillargeon, 1982).

Por otro lado, la emergencia de la habilidad para ordenar sucesos en una secuencia temporal se puede localizar ya en el período prelingüístico. De hecho, en el trabajo realizado por Piaget (1954) sobre el período sensoriomotor, él observó el desarrollo de relaciones temporales en niños de 18 meses y describió las comprensiones más tempranas de los niños de las relaciones temporales principalmente en términos de seriación temporal. Al dominar la seriación, los niños discriminan perceptivamente, imitan y posteriormente recuerdan una sucesión de cambios. Mediante la descripción de la seriación temporal, Piaget intentó explicar el modo en que los niños pasan de un tiempo personalizado y centrado en la acción a un sentido del tiempo de mayor amplitud y más objetivo.

Además, en la transición desde modos de pensamiento sensoriomotores a otros representacionales, Piaget habló ya de la noción de presente expandido en el sentido de que los niños se vuelven capaces de mantener imágenes ordenadas en su memoria que se corresponderían, de algún modo, con el orden de sucesos y estados precedentes. E incluso sugirió la idea de la existencia de un presente expandido que incluiría el futuro inmediato cuando habló de la intencionalidad práctica y de las anticipaciones tempranas de los niños pequeños. 
Por otro lado, a partir de los resultados obtenidos en varios estudios recientes (Bauer y Mandler, 1989; Day, Stein y Trabasso, 1979; Mandler y Johnson, 1977; Stein y Glenn, 1979) sabemos que incluso los niños de tan sólo 4 años tienen poca dificultad en recordar historias en el orden exacto de la secuencia del texto si dicha secuencia corresponde con la forma canónica esbozada en las gramáticas. Esos datos contradicen las afirmaciones de Piaget (1923/1960) de que los niños preoperacionales no tienen la capacidad de hacer uso de las relaciones causales o de mantener el orden temporal correcto de la información del texto. De hecho, en un intento de comprender la razón de que Piaget hiciera esas afirmaciones, Stein y Trabasso (1981) reanalizaron los datos de Piaget (1923/1960) y encontraron muy pocos errores de ordenación en el recuerdo de los cuentos realizado por los niños (Stein y Glenn, 1982).

En un sentido diferente, la mayor parte de la investigación sobre la ontogenia del concepto de tiempo y de la estimación del tiempo, ampliamente estudiada desde el trabajo pionero de Piaget (1946), comienza después de la etapa infantil, cuando el ser humano empieza a dominar las herramientas simbólicas y lingüísticas. Existe un gran vacío informativo sobre los precursores de esas habilidades cognitivas y simbólicas en la primera infancia.

Las razones de que el desarrollo temprano del cronometraje y de la estimación de la duración temporal haya sido infra-investigado son tanto teóricas como metodológicas. Teóricamente, los resultados de numerosos estudios (Fraisse, 1948; Fraisse y Orsini, 1958; Goldstone y Goldfard, 1966) pusieron de manifiesto que los seres humanos no podían percibir y estimar la duración hasta que dominasen los conceptos generales de tiempo y medida, y que el período de los 6 a los 8 años es una etapa de transición en varios sentidos. Sin embargo, hay «modos de adaptación al tiempo» (Fraisse, 1982), presentes antes del dominio de la noción de tiempo, que no presuponen pensamiento y conocimiento conceptual. Son capacidades discriminativas básicas relacionadas con la percepción del tiempo que incluyen la habilidad para detectar la frecuencia temporal y diferencias en duración. Además, existen una serie de capacidades perceptivas de más alto nivel que dependen del dominio temporal para conseguir información sobre el entorno, entre las que destacan el agrupamiento perceptivo, la segregación perceptiva y la percepción de los objetos. En esta línea de trabajo, Demany, McKenzie y Vurpillot (1977), así como Washburn y Cohen (1984) encontraron que los niños de 3 a 5 meses son capaces de percibir secuencias rítmicas auditivas, aunque la capacidad para percibir las propiedades globales de una estructura temporal compleja no aparecen hasta el final del primer año de vida (Morrongiello, 1984). También se ha encontrado que los niños muy pequeños son capaces de responder a variaciones en las propiedades temporales de la estimulación visual, tanto estática (Gardner y Karmel, 1984), como dinámica (Kaufmann, Stucki y Kaufmann-Hayoz, 1985; Mendelson, 1986). De igual modo, recientemente se ha constatado que los niños pequeños detectan la información temporal inherente en los sucesos multisensoriales (Lewkowicz, 1985a, 1985b, 1986, 1988a, 1988b).

Otra razón de la escasez de estudios sobre el desarrollo a una edad muy temprana de la capacidad para cronometrar la conducta y estimar la duración es de tipo metodológico ya que resulta difícil aplicar a edades tempranas los métodos psicofísicos tradicionales puesto que dependen, en gran medida, de técnicas verbales, e incluso algunos de ellos, como la estimación verbal y la producción, exigen conocimiento de las unidades convencionales de tiempo. Pero, 
aun a pesar de esto, existen trabajos (Crowder y Hohle, 1970; Friedman, 1977) en los que, mediante el empleo de tareas de reproducción, se ha demostrado que, con suficiente práctica y retroalimentación, los niños de 4 años pueden reproducir intervalos de tiempo. Para paliar la problemática metodológica mencionada, se están investigando actualmente (Pouthas, 1981, 1985) distintos tipos de paradigmas de condicionamiento operante que pueden resultar más apropiados para explorar las capacidades de estimación temporal en niños muy pequeños. Esta línea de investigación ontogenética puede arrojar información sobre el modo en que formas nuevas de conducta temporal se sobreimponen sobre aquellas observadas previas a la aparición de la función simbólica y de las representaciones cognitivas del tiempo.

Finalmente, hasta fechas recientes se había pensado que el tiempo lógico, entendido como la posibilidad de correlacionar un suceso dado con una posición única en un continuo temporal (Friedman, 1978), no se desarrollaba hasta los 8 años. Sin embargo, Weist (1989) en su aproximación indirecta a este problema mediante la realización de inferencias sobre lo que los niños de edades entre el año y medio y los 4 años y medio conocen de la temporalidad por lo que dicen, ha encontrado una secuencia evolutiva de cuatro sistemas temporales. En dicha secuencia, cada sistema contiene una serie de conceptos temporales interrelacionados que ponen de manifiesto que, a medida que los niños adquieren el lenguaje, las configuraciones de los conceptos de tiempo que pueden comprender y producir se van haciendo más complejos de forma progresiva.

A pesar de que la falta de información sobre el desarrollo cognitivo de la temporalidad en los primeros años de la vida del niño no es tan grande como se sostenía hasta mediados de la década de los 80 , déficit paliado gracias a la investigación realizada muy recientemente, todavía quedan muchas cuestiones no resueltas. Por ejemplo, aún se desconoce cómo interaccionan los ritmos bioconductuales intrínsecos con las capacidades de procesamiento temporal y la forma en que esa interacción recíproca contribuye al desarrollo de los aspectos motores, lingüísticos, afectivos y sociales de la conducta de los niños muy pequeños. Por todo ello, el conocimiento sobre los factores temporales en los primeros años de vida del ser humano sigue resultando insuficiente, sobre todo cuando se compara con el bagaje de conocimientos que poseemos sobre las edades posteriores.

\section{DE LOS 5 A LOS 12 AÑOS}

La idea de que en el ser humano no era posible que se generase conocimiento temporal significativo hasta la obtención de las herramientas simbólicas y lingüísticas ha determinado que, mayoritariamente, la edad crítica en la que se comenzaban a investigar los aspectos temporales se situara en torno a los 5 años de edad. De ahí que, tradicionalmente, los investigadores orientados de forma evolutiva situaran en ese punto el límite inferior de edad considerado y se extendieran no más allá de la adolescencia por considerar que esos eran los marcadores del sector evolutivo de su interés. Esa limitada consideración sobre el desarrollo ha traido consigo la proliferación, sobre todo hasta el comienzo de la adolescencia, de estudios en torno a la amplia gama de problemas temporales que a continuación se abordan.

Antes de proceder a comentar el desarrollo de esos núcleos temáticos sobre 
el tiempo, permítasenos una aclaración. El período de tiempo que va de los 5 a los 12 años no supone un intervalo homogéneo en lo que al conocimiento temporal conseguido por el niño se refiere. En términos piagetianos, en dicho intervalo aparecería incluido el final del período preoperacional y todo el período de las operaciones concretas. En los estudios sobre el desarrollo del tiempo se ha visto que, de un modo general, habría un período en torno a los 4-5 años caracterizado por un conocimiento intuitivo de la temporalidad, un período de transición (6-7 años) y, finalmente, aparecería el dominio cognitivo de tipo operacional concreto que se extendería de los 8 a los 12 años de edad, aproximadamente. Si no seguimos la partición propuesta por Piaget en nuestra organización del trabajo es porque nos interesa más ilustrar la progresión continua que sigue el desarrollo de cada aspecto temporal en lugar de comentar en bloque el estado de todas las posibles nociones temporales en cada período evolutivo al estilo piagetiano.

\section{A. El desarrollo del concepto de tiempo}

La bibliografía evolutiva más tradicional sobre el tiempo se ha referido al «concepto de tiempo» como si realmente existiera una unidad. Tal alusión, aun siendo atractiva, resulta totalmente ilusoria puesto que lo que hay son diversos aspectos del tiempo que se resisten a ser aunados en un concepto único. En esta situación, en absoluto resulta extraña la inexistencia de una única teoría sobre el desarrollo del concepto de tiempo. Al modelo postulado por Piaget (1946) le han seguido un gran número de propuestas alternativas para modelar los cambios que se producen en el conocimiento que el niño posee sobre el tiempo.

\section{La teoría piagetiana}

El análisis realizado por Piaget (1946) sobre la evolución del concepto de tiempo refleja las premisas fundamentales de su modelo general del desarrollo. De ese modo, describió la existencia de tres etapas generales en la construcción por parte de los niños de los conceptos de tiempo: una etapa intuitiva, una segunda de transición y una tercera operacional concreta.

De acuerdo con Piaget $(1969,1979)$, tras la conexión estrecha existente en el plano puramente sensoriomotor que vincula la construcción de la primeras relaciones temporales con la coordinación de los movimientos - tanto en el interior de los esquemas que caracterizan las costumbres y las «reacciones circulares» como en la asimilación recíproca de los esquemas que desembocan en el grupo práctico de los desplazamientos y en la noción de objeto permanente-, las nociones intuitivas de tiempo, espacio y velocidad emergen hacia los 4 ó 5 años y se van construyendo progresivamente hasta alcanzar la lógica newtoniana hacia los 9 años de edad.

El niño intuitivo no distingue entre la dimensión abstracta de tiempo y los sucesos que ocurren durante ese tiempo. El tiempo en esa etapa adquiere su significado a través de los movimientos, los sucesos o las acciones, de tal modo que no se concibe como una dimensión unitaria, continua o uniforme, sino que cada suceso tiene su propia escala de tiempo con su ritmo particular.

La dificultad del niño intuitivo de abstraer el tiempo de los sucesos que lo llenan proviene, según Piaget, de su incapacidad para tomar en cuenta o coordinar diferentes velocidades. Puesto que la noción de velocidad no es muy diferente de la del ritmo del tiempo, cuando dos sucesos van a diferentes velocidades 
el niño es incapaz de situarlos en una escala de tiempo común y unitaria. De este modo, el niño pequeño trata los dos sucesos o movimientos como si fuesen una acción simple ignorando sus diferencias en velocidad. Por lo tanto, la duración se relaciona directamente con el resultado de la acción.

Además, puesto que las imágenes que el niño intuitivo tiene del tiempo están centradas en la acción o limitadas por el movimiento, en esta etapa el niño no es capaz de inferir las relaciones lógicas entre los diversos conceptos temporales de sucesión, simultaneidad y duración. Para el niño de 4 ó 5 años, dos sucesos pueden comenzar y acabar a la vez y, no obstante, durar diferentes duraciones. Además, uno de los dos sucesos que comenzaron a la vez puede pararse después del otro, pero a pesar de eso consumir menos tiempo.

El progreso desde las nociones intuitivas sobre el tiempo a las nociones de transición de la etapa piagetiana intermedia se debe a la articulación del concepto de velocidad. La velocidad, en su etapa intuitiva, se reduce a experiencias de alcanzar o adelantar. Con la conciencia de diferencias en velocidad de sucesos sincrónicos, incluso aunque la velocidad se deduzca solamente de alcanzar o adelantar, los niños empiezan a ser capaces de distinguir entre el tiempo en el que los sucesos tuvieron lugar y sus productos (p.e., distancia recorrida). La distinción entre tiempo y espacio está, no obstante, limitada en esta etapa de transición o a la duración o a la sucesión, esto es, los niños pueden comprender que el concepto de duración es independiente del de distancia cubierta, pero no distinguen «antes» y «después» en tiempo de «antes» y «después» en espacio. No se comprende todavía la relación lógica entre duración y sucesión.

La etapa de las operaciones concretas se caracterizaría, siempre según Piaget, por dos logros principales. Por un lado, los niños consiguen comprender la necesaria relación lógica entre sucesión y duración y, por el otro, son capaces de coordinar tiempo, velocidad y distancia. No sólo se harán conscientes de la relación entre cada par de esos conceptos, sino que además comprenden sus interrelaciones totales. De ese modo, son capaces de tratar con esos conceptos dentro del esquema logicomatemático general de la etapa de las operaciones concretas.

La ley algebraica que relaciona las tres dimensiones (velocidad = distancia/tiempo) es acorde con la teoría física de la mecánica newtoniana, y, en la práctica diaria, la física newtoniana continúa dando a los adultos la única lógica absoluta y autoevidente del mundo físico. El concepto einsteniano de tiempo se logra sólo con entrenamiento especial en física y, por lo tanto, no puede verse como el estado final natural del desarrollo del concepto de tiempo. Muchos adultos no adquieren nunca ese tipo de conocimiento relativista sobre el tiempo. Pudiera ocurrir que esta última comprensión fuese en el futuro de tan amplio alcance y tan autoevidente para el adulto como el conocimiento de que la tierra no es plana, pero en el momento en que Piaget trabajó este tema no era así, por lo cual él consideró que el dominio alcanzado en la etapa operacional concreta en relación con los conceptos de tiempo suponía una lógica madura por parte del niño, similar a la puesta de manifiesto por los adolescentes y por los adultos.

Resumiendo, tanto Piaget como sus seguidores sostienen que el niño debe construir el concepto de tiempo coordinando dimensiones relacionadas en una estructura algebraicà. Los estados inmaduros de conocimiento serían el resultado de deficiencias lógicas en dicha estructura. De acuerdo con esta teoría, los niños son capaces de resolver los problemas de sucesión y duración relativa solamente cuando adquieren las operaciones que les permiten la estructuración 
lógica de las relaciones temporales, generalmente alrededor de los 8 6́ 9 años de edad. Antes de esa edad los niños no podrían tener un concepto de tiempo independiente de la velocidad y de la distancia puesto que se piensa que no pueden distinguir entre esas dimensiones.

Es por eso que el paradigma de tarea empleado por Piaget, y por muchos otros después de él (p.e., Acredolo y Schmid, 1981; Siegler y Richards, 1979), era uno de elección en el que se incluían las señales de velocidad y de distancia. Se presentaba a los niños una tarea de comparación lineal entre tiempos en la que se incluían dos pequeñas figuras que seguían vías paralelas. Dichas figuras comenzaban a la vez en el mismo sitio, pero una de ellas se movía durante más tiempo mientras que la otra iba más deprisa, hacía un recorrido mayor y se detenía por delante de la anterior. El hallazgo de Piaget de que los niños muy pequeños consideraban que la figura que había recorrido la mayor distancia era también la que había empleado un tiempo mayor le llevó a considerar que el niño confundía las señales temporales con una señal espacial de distancia. $\mathrm{Ha}$ cia los 6 años según Piaget, los niños serían ya capaces de separar en alguna medida, aunque no totalmente, las señales espaciales de las temporales, pero habría que esperar hasta el momento en que se adquiriesen las operaciones concretas (7-8 años) para poder observar un dominio completo del concepto de tiempo caracterizado por una coordinación de todas las relaciones espaciotemporales necesarias.

Lovell y Slater (1960) en su réplica y extensión del trabajo realizado por Piaget sobre comprensión temporal humana dieron diferentes problemas a los mismos niños en vez de ofrecérselos a niños diferentes. Aunque obtuvieron apoyo general a los resultados obtenidos por Piaget, dichos autores encontraron que algunos problemas temporales podían ser resueltos por niños muy pequeños, mientras que otros eran muy difíciles incluso para niños de 9 años. La interpretación ofrecida por Lovell y Slater insistía en que algunas relaciones lógicas entre señales espaciales y temporales se dominan antes que otras.

\section{La teoría de los factores interfirientes}

En contraste con la teoría piagetiana que enfatiza la lógica inmadura o madura del niño, la teoría de los factores interfirientes se centra en los aspectos de los problemas temporales que impulsan al niño a equivocarse. Levin (1977, 1979, 1982, 1989) y sus colaboradores (Levin, Gilat y Darom, 1980; Levin, Israeli y Darom, 1978; Levin y Wilkening, 1989) han mostrado que si se eliminan las señales interfirientes de velocidad y de distancia, los niños de tan sólo 5 años son conscientes de la relación lógica que existe entre duración y sucesión. A partir de la obtención de estos resultados se deriva que, contrariamente al postulado piagetiano, se puede investigar significativamente la comprensión temporal sin entremezclar las señales de tiempo con las de velocidad y de distancia. Por lo tanto, no hay uno sino varios niveles de comprensión de la duración, con lo cual el concepto de duración no puede derivar de la coordinación de las velocidades como argumentaba Piaget.

Además, Levin explica el fallo de los niños pequeños en juzgar la duración en los problemas del tipo posición-velocidad-distancia basándose en la idea de que las características de la tarea - p.e., relevancia de las señales no temporalesy las altas demandas impuestas sobre la memoria impiden la extracción de información de tiempo crítica. Por esta razón, dicha autora propone un modelo 
sobre la influencia de factores de interferencia en los juicios de duración de los niños.

Una variante del acercamiento de las variables interfirientes es la teoría sobre la integración de la información en la que se considera que la capacidad de procesamiento de la información determina la habilidad del niño para coordinar variables temporales y espaciales. Dicha variante fue propuesta por Wilkening $(1979,1981)$ quien, utilizando un paradigma derivado de la teoría de integración de la información de Anderson (1981), ha investigado la capacidad de los niños para cuantificar e integrar la información de duración, velocidad y distancia. Wilkening encontró que los niños de preescolar son capaces de extraer e integrar la información de cualesquiera de dos de esas dimensiones para juzgar una tercera, aunque utilicen una regla sustractiva para inferir el tiempo restando la velocidad de la distancia. Se piensa, entonces, que las reglas de integración particulares implícitas en los juicios infantiles de duración cambian con el desarrollo hasta llegar a la regla de cociente normativa y propia de los adultos en la que el tiempo se obtiene a partir del cociente de la distancia por la velocidad.

Tanto la teoría de Piaget como la de los factores interfirientes fallan en considerar todos los aspectos del desarrollo de la comprensión del tiempo de modo simultáneo. Los teóricos piagetianos se centran en las características de quien resuelve el problema sin tener en cuenta las demandas exigidas por la tarea; no contestan a la pregunta de por qué los niños son capaces de resolver algunos problemas temporales pero no otros. Los teóricos de los factores interfirientes hacen hincapié en las demandas de la tarea de juicio temporal e insisten menos en la persona que resuelve el problema; en el mejor de los casos, son imprecisos respecto de la cuestión sobre por qué los niños más mayores pueden resolver problemas de tiempo que los niños más pequeños son incapaces de resolver correctamente. Parecería beneficiosa, entonces, una aproximación mejor equilibrada que considerase no sólo las demandas de la tarea y las habilidades de quien resuelve el problema, sino también la interacción entre esos dos aspectos.

\section{La teoría de las relaciones conceptuales}

Por medio de esta teoría (Richards, 1982; Siegler y Richards, 1979) se postula que la comprensión completa de un concepto como el de tiempo requiere conocer no sólo las relaciones entre ese concepto y otras dimensiones, sino también las propiedades únicas del concepto. El primer tipo de conocimiento exige una comprensión de las correlaciones imperfectas entre el concepto clave y otros conceptos, así como el conocimiento de las condiciones bajo las cuales esas correlaciones son moderadas, débiles y fuertes. El segundo tipo de conocimiento constituye la parte única del concepto, no relacionado con, ni descriptible en términos de cualquier otro concepto.

Lo que cambiaría, entonces, evolutivamente, sería el aprendizaje de las relaciones entre el concepto específico y los conceptos relacionados con él. Este proceso de aprendizaje supone tanto separar conceptos similares como unir conceptos diferentes, o sea, establecer las condiciones bajo las que la relación aparece y desaparece y el mecanismo mediante el que operan dichas relaciones. Se supone que las relaciones se establecen y se refinan mediante la asociación repetida, de tal modo que se podría esperar que las relaciones basadas en asociaciones encontradas frecuentemente se instaurasen antes que las fundamentadas en asociaciones menos frecuentes. 
Un aspecto final de esta teoría trata sobre la unión entre la comprensión de un concepto por parte del niño y su desempeño en una tarea dada. Este último dependería solamente del emparejamiento entre las relaciones conceptuales conseguidas por el niño y aquellas presupuestas por la tarea. El completo dominio del concepto se conseguiría cuando se comprendiese completamente cada relación perfecta o imperfecta con otro concepto. Sin embargo, para la mayor parte de los conceptos complejos, ese criterio de comprensión es demasiado restrictivo. Hay pocos casos reales en los que se puedan conocer todas las relaciones entre un concepto determinado y otros conceptos, así como todas las condiciones bajo las que esas relaciones se aplican. De ahí que se utilice la comprensión de las relaciones a un nivel suficiente como para utilizar el concepto bajo la mayor parte de las condiciones que probablemente puedan encontrarse como el criterio para definir el dominio de dicho concepto.

En la aplicación específica de esta teoría al concepto de tiempo se asume que el concepto de tiempo estaría constituido por los aspectos únicos del tiempo, así como por las relaciones entre el tiempo y la distancia, entre el tiempo y la velocidad, entre el tiempo y el tamaño de objetos crecientes, etc. Por lo tanto, el desarrollo del concepto de tiempo se compondría primariamente mediante el establecimiento de esas relaciones y de las condiciones bajo las que dichas relaciones son aplicables. Por ejemplo, distancia y tiempo son directamente proporcionales, pero sólo bajo condiciones de velocidad uniforme. La ejecución del niño en problemas de tiempo dependerá del emparejamiento entre la comprensión del niño y las relaciones presentes en el problema. Siguiendo con el ejemplo, un niño que cree que distancia y tiempo son siempre directa. mente proporcionales resolverá bien los problemas en los que esa relación se mantenga, pero mal los problemas que involucran las excepciones a esa regla.

Para demostrar lo acertado de las propuestas efectuadas por la teoría de las relaciones entre conceptos se ha utilizado la metodología de evaluación de reglas bajo los supuestos de que los niños comprenden los conceptos en forma de reglas específicas y de que esas reglas se pueden discriminar mediante la construcción de problemas cuidadosamente diseñados. Dicha metodología involucra primero la formulación de modelos de reglas que los niños podrían utilizar para ejecutar varias tareas y luego formular diferentes tipos de problemas que permitan la discriminación entre las reglas. Es una metodología reveladora y fiable porque se ha demostrado que es capaz de desvelar las estrategias que los niños de diferentes edades utilizan para resolver distintos tipos de problemas que suponen el dominio de las operaciones concretas o de las operaciones formales, a la vez que permite efectuar puntuaciones totalmente fiables puesto que se apoya en simples respuestas no verbales como datos básicos.

La regla que el niño utiliza refleja la interacción entre las características de la tarea y el conocimiento que el niño posee. De ese modo, esta metodología de evaluación de reglas combina la naturaleza específica de la tarea de los estudios sobre los factores interfirientes con el énfasis en el nivel de comprensión del niño presente en los estudios de tipo piagetiano.

De todas maneras, los distintos modelos propuestos sobre el conocimiento del tiempo no son netamente contradictorios, sino que dependen de diferentes definiciones del concepto de duración y de variados criterios metodológicos para su logro. En este sentido, se han cuestionado los criterios utilizados por Piaget y sus seguidores para atribuir la existencia de conocimiento temporal en los niños por la utilización que hacían de tareas con señales muy distintivas (p.e., 


\section{1}

los problemas de posición-distancia-velocidad) y de un método para diferenciar etapas basado en una combinación de juicios y explicaciones.

Reaccionando a esa situación, la investigación más reciente ha buscado la simplificación de las tareas utilizadas para conseguir información sobre el patrón temporal evolutivo básico, imposible de ser obtenida a partir de las tareas más complejas de tipo piagetiano. También se han variado los criterios de respuesta empleados en un intento de reducir las demandas impuestas al niño, eliminando, en unos casos, las explicaciones del juicio exigidas (Richards, 1982) y evitando, en otras ocasiones, tanto las explicaciones como los juicios relativos pidiendo al niño estimaciones directas de las dimensiones (Wilkening, 1981).

\section{B. El desarrollo de la perspectiva temporal}

Las nociones de pasado, presente y futuro son centrales al modo en que organizamos nuestra vida y a la forma en que pensamos sobre los sucesos, las experiencias y nosotros mismos. A lo largo de su desarrollo, los niños han de dominar cada una de las distinciones conceptuales incluidas en el modelo temporal mantenido en nuestra cultura. Entre esas características clave se incluyen: (a) la secuenciación temporal de dos sucesos uno en relación al otro; (b) el pasado y el futuro como categorías mutuamente exclusivas; (c) el doble significado del presente, como punto de referencia y como categoría de la experiencia que se puede expandir; (d) los límites cambiantes entre el pasado y el presente, así como entre el presente y el futuro; (e) la naturaleza continuamente cambiante del presente y los contenidos cambiantes de cada una de las categorías temporales de pasado, presente y futuro; (f) la necesidad de descentrarse y de considerar un tiempo de referencia diferente del propio presente inmediato de uno.

El lenguaje es el medio a través del cual se transmiten las nociones sobre sucesos pasados y futuros, ideas que no tienen una realidad física, externa e inmediata. El lenguaje proporciona las estructuras para pensar y comunicarse. Esas estructuras lingüísticas pueden influir los procesos psicológicos involucrados en conceptualizar los sucesos pasados y futuros (Harner, 1982). Las variedades de lenguaje que codifican nociones de tiempo no-presente han variado sustancialmente en las distintas sociedades, así como dentro de cualquier comunidad lingüística particular. La mayoría de los lenguajes codifican nociones de tiempo presente, pasado y futuro (Bull, 1971), aunque los diferentes idiomas varían entre sí en las formas que ofrecen y en las distinciones que realizan. Hay un sistema lingüístico elaborado relacionado con las nociones de tiempo que los niños deben dominar gradualmente. En este sentido, los investigadores han estudiado cómo consiguen comprender los niños el «lenguaje del tiempo».

Los métodos utilizados para conseguir información de los niños han sido de dos tipos principales: primero, se ha analizado el lenguaje espontáneo y elicitado de los niños para evaluar su dominio de formas lingüísticas marcadas temporalmente en diferentes contextos; y segundo, se les han formulado preguntas sobre acciones y se han registrado sus respuestas siendo consideradas éstas como indicadores de su comprensión lingüística y cognitiva de referencia temporal. Además, el lenguaje temporal de los niños y sus conceptos de tiempo se han comparado con las normas de los adultos y se han analizado sus errores para obtener información sobre la naturaleza del proceso de adquisición (Harner, 1982).

No está claro de distintos estudios realizados (Clark, 1971; Cromer, 1971; 
Estaun-Ferrer, 1991; Stern y Stern, 1907) a qué tipo de sucesos aprenden a referirse antes los niños, si a sucesos pasados o a eventos futuros, o a ambos tipos al mismo tiempo. Lo que sí parece asentado es que su adquisición del sistema comienza con verbos marcados temporalmente, después de los cuales aparecen los adverbios referidos al no-presente (p. e., ayer, mañana). Por lo que se refiere a la naturaleza del uso temprano de los verbos en tiempo pasado (Antinucci y Miller, 1976; Bloom, Lifter y Hafitz, 1980; Bronckart y Sinclair, 1973; Sachs, 1979; Smith, 1980), los significados que los niños les adjudican son menos complejos que los correspondientes a la noción de pasado de los adultos. Parece ser que al principio existe un interjuego continuo entre el tiempo subjetivo y el objetivo puesto que los niños utilizan el pasado para referirse a un pasado-en-elpresente dado que su motivación fundamental sería hablar sobre los sucesos inmediatos que tienen significado actual.

No obstante, una vez que los sistemas conceptuales y lingüísticos se dominan, la experiencia subjetiva no decae totalmente. La investigación ha encontrado que factores tales como la clase social, la edad, el sexo y la raza influyen la perspectiva temporal individual (Moerk, 1974) haciendo que, aun poseyendo el mismo sistema objetivo, nuestras experiencias individuales nos conduzcan a orientaciones subjetivas distintas hacia el tiempo y el mundo.

Por otra parte, distinguir el pasado y el futuro como dominios mutuamente excluyentes es conseguido relativamente pronto por la mayor parte de los niños, particularmente cuando la referencia lingüística se hace con verbos pasados o futuros, o cuando los adverbios antes o después se usan para ordenar un suceso en relación con un punto de referencia presente, tal y como encontraron Carranza, Brito de la Nuez y Torrecilla (1984) con niños de entre 4 y 6 años ó Harner (1980) cuando preguntó a niños de entre 3 y 7 años. Sin embargo, estos últimos niños tenían menos claros los límites presente-pasado y presente-futuro.

Por lo que respecta a la descentración temporal, esto es, la habilidad para cambiar el tiempo de referencia de uno desde el momento en que se está hablando a otro punto en el tiempo, Miller (1978) y Smith (1980) consideran que los niños pequeños no suelen ser capaces de coordinar el tiempo de los sucesos, el del discurso y un tiempo de referencia que difiera del tiempo del discurso. Sería hacia los 7 años cuando se consigue seleccionar el referente correcto en la mayor parte de los casos (Harner, 1980).

También se ha investigado la forma en que diferentes idiomas favorecen o dificultan el dominio del lenguaje del tiempo; la mayoría de los trabajos en este sentido han tomado como objeto de estudio las peculiaridades del inglés (Harner, 1975). Un último tema debatido ha sido la asimetría del pasado y del futuro tanto en el dominio de la experiencia personal y práctica como en el dominio de la estructura lingüística (Carranza, Brito de la Nuez y Torrecilla, 1984).

A medida que los niños van dominando las distinciones conceptuales y lingǘsticas y las coordinan con su experiencia práctica de los tiempos pasado y futuro, también van ampliando su comprensión de la extensión del pasado y de la secuenciación de los sucesos, y se vuelven más capaces de cuantificar el tiempo y de imaginar un futuro nuevo y distante. Comprender y comunicarse claramente sobre las relaciones básicas de pasado, presente y futuro es un logro importante en sí mismo, además del fundamento para el desarrollo potencial de un mayor cuerpo de conocimiento temporal. 


\section{El desarrollo de la medida del tiempo}

Una de las cuestiones centrales en la investigación evolutiva reciente sobre el tiempo e implícita en las teorías sobre el concepto de tiempo ha girado en torno al momento en que los niños son capaces de tratarlo como una dimensión cuantificable. Durante décadas, el impacto de la teoría piagetiana ha impedido el adecuado abordaje de este tema debido a su adhesión al paradigma de elección por cuanto, bajo el supuesto de que la capacidad para cuantificar la duración era un logro tardío en el curso del desarrollo, se consideraba que los niños pequeños únicamente podían tener un concepto ordinal del tiempo.

Pero hallazgos recientes convergen hacia la conclusión de que los niños de 5 años pueden concebir el tiempo como una entidad cuantitativa. Además de los conceptos métricos temporales encontrados por Wilkening (1981) en relación con la velocidad y la distancia, otros estudios han encontrado que los niños pueden cuantificar las duraciones basándose en información temporal pura sin involucrar las informaciones de distancia y velocidad (Levin, Wilkening y Dembo, 1984; Richie y Bickhard, 1988; Wilkening, Levin y Druyan, 1987).

Por ejemplo, en el estudio realizado por Richie y Bickhard (1988) se encontró que niños de entre 3 y 6 años eran capaces de resolver problemas que son lógicamente imposibles de resolver sobre la base de la información no temporal, lo cual indicaría que los niños pequeños tienen una experiencia perceptiva de la duración separada de la que se deriva de forma inferencial del conocimiento no temporal y que pueden utilizar ese conocimiento perceptivo para resolver problemas de duración relativa.

Hay que matizar, sin embargo, que la cuantificación que los niños pueden llevar a cabo sobre el tiempo no aparece en cada tipo de tarea a la misma edad, p.e., cuando los sucesos se presentaban sucesivamente, la regla de integración algebraica correcta se empleaba mayoritariamente a los 6 años, la misma edad a la que se juzgaba adecuadamente la duración de sucesos simples, pero cuando se han presentado sucesos parcialmente simultáneos, se ha encontrado un predominio de las reglas cualitativas para juzgar el tiempo a los 10 años de edad.

Además, la cualidad de los juicios temporales infantiles no sólo varía con la estructura del problema, sino también con las estrategias empleadas para medir el paso del tiempo, de modo que cuando se cuenta, aumenta la fiabilidad de los juicios y la probabilidad de conseguir una regla correcta de integración. Pero contar de forma espontánea supone un alto nivel de abstracción que algunos niños de 5-6 años no pueden conseguir por lo que se ven obligados a inventarse métodos idiosincráticos para medir el tiempo, tales como imitar el sonido de un reloj o intentar acumular el tiempo imaginando escenas. Al margen de la eficiencia con que los niños pequeños consigan medir el tiempo, queda claro que son capaces de encontrar métodos para medirlo y juzgarlo, contrariamente a lo que pensaba Piaget (1946).

\section{El desarrollo del tiempo convencional y de la estructuración temporal}

Los sistemas del tiempo convencional plantean interesantes cuestiones evolutivas dado su significado ecológico, sus ricas estructuras y sus especiales demandas representacionales. No obstante, la bibliografía sobre este tema es muy limitada.

La información más asentada de que se dispone sobre este tema, obtenida 
pidiendo a los niños que digan el día o el mes actual o bien solicitando el orden de los diferentes elementos (Estaun-Ferrer, 1982; Friedman, 1978), es indicativa de la edad a la que los niños conocen el orden de los días de la semana y de los meses del año, que suele ser entre los 5 y los 8 años de edad. Las propiedades estructurales cíclicas del orden y de la recurrencia son difíciles de integrar por los niños antes de los 8 ó 9 años, y ciertos tipos de problemas que suponen la coordinación de series temporales dispares se dominan también en torno a esa edad (Friedman, 1991). La distinción entre duraciones naturales y arbitrarias en los sistemas de tiempo convencional aparece en las explicaciones de los niños a los 11 años y en sus juicios hacia los 9 años.

Menos se sabe, no obstante, sobre la forma en que se adquiere dicho tipo de conocimiento, esto es, sobre los modos de representación que subyacen el conocimiento temporal infantil a diferentes edades y cómo cambian las representaciones con el desarrollo (Friedman, 1982). Recientemente, Friedman (1986, 1989) ha propuesto un modelo de etapas según el cual en una primera etapa no habría realmente representación del orden de los elementos, sino que solamente se sabría asociar una etiqueta temporal con un suceso dado. De acuerdo con este modelo, la primera codificación del orden temporal probablemente se realice en forma de representaciones del tipo de lista verbal dada la frecuencia con que se enseña a los niños los días y los meses como listas para recitar. Una última etapa supondría la representación del orden de los días y de los meses por medio de imágenes, formato que resulta más eficiente que el anterior para la extracción de determinadas relaciones de orden. Los resultados obtenidos por Friedman $(1986,1989)$ indican que, efectivamente, las representaciones que utilizan imágenes se desarrollan más tarde que las basadas en listas verbales. Aquellas constituyen un logro superior en nuestro conocimiento sobre el mundo temporal de compleja estructura en que nos encontramos.

La crítica que se puede realizar a este tipo de trabajos es que los estudios empíricos que se han realizado describen la ejecución de los niños a diferentes edades, pero no indican cuáles son los procesos que conducen a los cambios, lo cual impide conocer si los cambios evolutivos se deben a transiciones cognitivas generales - como por ejemplo en el tipo de operaciones o en la capacidad de procesamiento disponible - o si son específicos a la historia de las operaciones llevadas a cabo sobre los contenidos particulares.

Puesto que un modelo general de etapas plantea ciertos problemas, quizás resulte más adecuado intentar explicar las edades y los cambios en términos específicos a los contenidos sobre los días y los meses. En este sentido, Friedman (1989) considera tres tipos de influencias sobre el desarrollo de las representaciones para un dominio particular, 1) la estructura formal del contenido, 2) las restricciones naturales de la representación y del procesamiento, y 3) la historia de las operaciones que una persona realiza sobre el contenido. El primer factor determina qué información debe ser captada en una representación, y la segunda restricción sobre el desarrollo de las representaciones radica en que los seres humanos aparentemente sólo pueden procesar la información de un modo limitado.

\section{E. Otras habilidades temporales}

Además de las capacidades mencionadas, existen otros aspectos relacionados con el tiempo cuya evolución resulta también de sumo interés. Entre ellos 
destaca el desarrollo de la babilidad para decir la hora, dominio que no ha recibido demasiada atención a pesar de su importancia práctica.

La habilidad para decir la hora por parte de los niños puede verse desde la perspectiva de las horas que son capaces de decir ó desde el punto de vista de las estrategias que utilizan. Con respecto a la secuencia evolutiva en que los niños identifican las distintas horas, parece ser que las horas en punto son las más fáciles de adquirir y, por lo tanto, las primeras que aparecen evolutivamente. Al conocimiento de las horas en punto le sigue el de las horas a intervalos de cinco minutos (p.e., las 3 y 25), y a éste, el de las horas con intervalos de un minuto (p.e., las 3 y 14) (Case, Sandieson y Dennis, 1986). A los 4 años aparecen los primeros signos de la habilidad para decir la hora y a los 10 años todavía sigue desarrollándose. La mayoría de los niños de 6 años identifican las horas en punto, las horas con intervalos de cinco minutos se dominan a los 7 u 8 años generalmente y las horas con intervalos de un minuto a los 8 ó 10 años de edad.

Pero a pesar de que se conoce el momento evolutivo en que los niños son capaces de decir la hora y el orden en que aprenden a hacerlo, se sabe mucho menos sobre cómo deciden la hora que es y cómo pasan de decir la hora mal a hacerlo correctamente. Se ha encontrado que para decir la hora de forma correcta, los niños emplean varias estrategias, no una sola. El uso de estrategias varía en cada niño y en cada tipo de hora, así como entre niños y entre horas diferentes (Siegler y Mcgilly, 1989). De todos modos, se necesita más investigación que aporte nuevos datos sobre las estrategias empleadas por los niños en este tipo de tarea temporal con un fuerte componente de reconocimiento perceptivo.

Otro aspecto temporal de interés se refiere al desarrollo del razonamiento causal, base fundamental para nuestra comprensión del mundo físico y, en particular, de los cambios en forma de desplazamientos y transformaciones que ocurren en él. En relación con este tema se ha visto que tras la posesión temprana por parte de los niños de los principios causales de determinismo, prioridad y mecanismo (en torno a los 3 años), a los 5 años ya escogen de forma consistente las causas sobre la base de información coherente con tales principios y no escogen los sucesos que podrían ser inconsistentes con dichos principios. Además, los niños más mayores son capaces de articular bien la explicación de sus elecciones, cosa que no hacen los más pequeños. En lo que se diferenciarían niños y adultos en este tema sería no en que sus modos de pensar sean fundamentalmente distintos, sino en que el pensamiento del niño depende más del contexto, de la complejidad y de las demandas verbales de la tarea, lo que limita la amplitud y flexibilidad con la que puede aplicar sus conocimientos.

La capacidad para hacer uso de las relaciones causales y para mantener el orden temporal correcto se ha investigado también en relación con la información de un texto, temática que se conoce como el desarrollo de los esquemas de las bistorias o la habilidad de los niños para contar cuentos. La cuestión que ha guiado la investigación en este terreno ha sido averiguar si los niños son capaces de utilizar su conocimiento de las restricciones temporales y causales entre los sucesos de una historia para construir o generar sus propias historias. La ejecución en tareas de generación exige un tipo de conocimiento algo diferente que el desempeño en tareas de recuerdo. En las tareas de generación, no se da a los niños la estructura organizada de los sucesos, sino que son ellos quienes tienen que crear su propia estructura.

En los estudios realizados sobre este tema (Stein y Glenn, 1982) se ha en- 
contrado que los niños exhiben un conocimiento de las secuencias de las historias similar al propuesto en las gramáticas, si bien aparecen diferencias evolutivas en el sentido de que los niños de 11-12 años construyen las secuencias de un modo más similar a la forma canónica de una historia que los niños de 7 años, cuyo conocimiento de la estructura de las historias es menos completo. El conocimiento de las precondiciones y de los sucesos habituales y normales que suceden en las secuencias de las historias también aumenta significativamente como una función del desarrollo (Stein y Trabasso, 1981), con lo cual en la actualidad no está claro si los niños pequeños construyen peor las historias en su orden canónico por utilizar una estragegia «inmadura» o si ese hecho se debe a un falta de conocimiento relevante del mundo real.

A los 10 ú 11 años, los niños también son más conscientes de la estructura de las historias y más sensibles a los efectos de diferentes variaciones en el texto realizadas por el hablante. A medida que aumenta su experiencia con los textos, los niños mayores pueden aprender a planificar historias con estructuras que difieren algo de la forma canónica. Además, aparecen diferencias evolutivas en algunos tipos de información categórica incluida en un episodio, siendo más probable que los niños mayores incluyan intentos y reacciones en sus historias.

La fuente de esas diferencias evolutivas permanece sin descubrir. Aunque se han lanzado diferentes hipótesis, el trabajo futuro en este área tendrá que ir encaminado a explicar las razones de esas diferencias en comprensión a lo largo del desarrollo humano.

\section{LA ADOLESCENCIA}

La mayor parte de la investigación evolutiva sobre la cognición temporal se ha detenido a la edad de 12 años, momento en el que, de acuerdo con los postulados piagetianos, comenzaría la etapa de las operaciones formales. Cuando se ha hecho una excepción a esa regla y se ha utilizado un grupo de edad superior, al considerar que el siguiente escalón en el desarrollo supone ya el dominio de la lógica abstracta y asumiendo implícitamente que no existirían diferencias en la forma de pensar de los adolescentes y de los adultos, generalmente se han empleado muestras de adultos. De ahí que existan tan pocos trabajos que dediquen atención sistemática a las peculiaridades del conocimiento temporal de los adolescentes.

El núcleo temático en el que, de forma específica,. existen investigaciones centradas en la adolescencia es el relativo a las perspectivas temporales. La cuestión de interés no ha sido cómo se domina el lenguaje y las ideas subyacentes en esa etapa, sino cuál es la percepción consciente que de su propio pasado y de su futuro tiene el adolescente, y cuáles son los factores que influyen esas percepciones (Harner, 1982). Los adolescentes son capaces de la auto-reflexión, de compararse a sí mismos con otros y de autoevaluarse. Esas capacidades en pleno desarrollo les posibilitan un cambio en intereses significativos. Sin embargo, algunos autores sostienen (Greene, 1986) que los cambios en la perspectiva temporal de los adolescentes no se deberían tanto a la emergencia del pensamiento operacional formal, sino a los factores contextuales relativos a la experiencia y al estatus a lo largo del curso vital.

El uso por parte de los adolescentes del lenguaje del tiempo apenas si se ha examinado en poblaciones normales puesto que se ha asumido que ellos, al igual que los adultos, pueden utilizar todo el espectro del sistema lingüístico 
para expresar las distinciones temporales. No resulta fácil encontrar normas establecidas para las percepciones subjetivas de los adolescentes, aunque se han dedicado algunos esfuerzos de investigación a este tema (Bortner y Hultsch, 1972; Bühler, 1968; Nurmi, 1989). Otros se han centrado en los efectos de variables tales como el sexo o la raza sobre las perspectivas futuras (Moerk, 1974; Seniger, 1988).

Parece ser que durante la adolescencia la conciencia del tiempo aumenta y, por lo tanto, hay mucha más planificación para el futuro de la que hubo durante la infancia. Junto a esto, cuando los adolescentes se hacen más mayores, sus planes de futuro se vuelven mucho más realistas. En este sentido, Moerk y Becker (1971) entrevistaron a adolescentes de entre 14 y 17 años y encontraron que, a mayor edad, aumentaba la edad óptima del matrimonio para los hombres, se incrementaba la amplitud de tiempo óptima para tener hijos y los adolescentes varones disminuían el número óptimo de hijos en una familia. Además, la aceptación de procedimientos para controlar la natalidad aumentaba dramáticamente a medida que incrementaba la edad de los adolescentes.

Moerk (1974) encontró que tanto el sexo como la pertenencia a grupos étnicos minoritarios afectaba las aspiraciones educativas, ocupacionales y económicas de adolescentes de 12 a 15 años, e, incluso, en un seguimiento longitudinal obtuvo que los cambios a lo largo del tiempo estaban también mediatizados por esas variables sociopersonales.

Cottle, Howard y Pleck (1969), examinando los efectos de la edad, del sexo y de la clase social sobre las percepciones del tiempo de los adolescentes, encontraron que a medida que la edad aumentaba — de 12 a 18 años-, había un cambio en la orientación temporal desde el recuerdo a la expectativa, que los adolescentes más mayores tenían un sentido mayor de las relaciones entre el pasado, el presente y el futuro y que los adolescentes de clase media estaban más interesados en el futuro distante mientras que los de clase social alta se interesaban más por las zonas más próximas al presente y por los aspectos históricos.

En cuanto a las diferencias por sexos, parece ser que los varones manifiestan más objetivos de tipo instrumental que las mujeres, las cuales se orientan más por las metas expresivas, al menos en ciertos contextos socioculturales (Seniger, 1988). Los hombres tienen una mayor orientación hacia el futuro mientras que las mujeres manifiestan una orientación más acusada hacia el presente (Seth, 1986). También se han encontrado diferencias interculturales en la orientación y organización del tiempo (Sundberg, Poole y Tyler, 1983; Vasquez, 1982).

Por lo que se refiere a otros aspectos del conocimiento temporal, las representaciones de los días y de los meses a base de imágenes se desarrollan entre el principio y la mitad de la adolescencia, con lo cual se hace posible extraer ciertas relaciones de orden de un modo más eficiente que basándose en listas verbales (Friedman, 1989). Sin que aún sepamos cuáles son los factores responsables de esa transición, lo que sí es seguro es que en la adolescencia poseemos las representaciones que nos permiten comprender dónde nos encontramos en nuestro mundo temporal de compleja estructura.

Por otro lado, Crepault $(1978 \mathrm{a}, 1978 \mathrm{~b}, 1979,1983)$ ha propuesto un modelo sobre el razonamiento cinemático de los adolescentes que describe en un lenguaje logico-matemático la evolución del conocimiento sucesivo y las reglas de transformación que permiten el paso de un estado al siguiente, así como el logro final de la regla de cociente normativa que relaciona los tres parámetros de distancia, velocidad y tiempo. 
Al final de la adolescencia, el razonamiento temporal que se posee presenta ya todas las características de la cognición temporal adulta.

\section{RESUMEN Y VALORACION}

El estudio del desarrollo del conocimiento humano sobre el tiempo desempeña un papel importante en cualquier consideración general sobre el desarrollo perceptivo y cognitivo puesto que el tiempo es una propiedad omnipresente de la experiencia sensorial dado que es información. Además, el tiempo es fundamental en la vida humana ya que sin la experiencia de tiempo no conoceríamos el significado de la vida. Cualquier teoría sobre la ejecución humana, y en particular, cualquier teoría que trate con la interacción entre el ser humano y un entorno de tarea estructurado, debe incorporar el hecho de que aquel es consciente de, o, al menos, utiliza las relaciones temporales de simultaneidad, orden, sucesión temporal y duración para tratar con los requerimientos del am. biente.

Si bien durante mucho tiempo se pensó que el conocimiento humano sobre la temporalidad exigía el dominio de las nociones simbólicas y lingüísticas, la investigación reciente sobre este tema ha puesto de manifiesto que los niños muy pequeños poseen habilidades temporales que les posibilitan el desarrollo de toda una serie de conductas afectivas, perceptivas, cognitivas, lingüísticas y sociales necesarias para conseguir una buena adaptación a su medio ambiente.

Con sólo unos meses de edad, los niños realizan frecuentemente movimientos estereotipados que ponen de manifiesto su temprana sensibilidad al ritmo y que pueden servir como medio de comunicación con las personas que los cuidan. Además, los niños pequeños se forman expectativas temporales en los intercambios comunicativos con sus cuidadores e incluso es posible que los movimientos de distintas partes de su cuerpo estén sincronizados con el discurso de los adultos. En el período prelingüístico se localiza ya la habilidad infantil para ordenar sucesos en una secuencia temporal y, poco después, se empiezan a adquirir las primeras ideas sobre la perspectiva temporal. En un sentido diferente, mediante el empleo de métodos apropiados se ha encontrado que, contrariamente a los postulados piagetianos, los niños muy pequeños poseen capacidades discriminativas básicas relacionadas con la percepción del tiempo entre las que se incluiría la habilidad para detectar diferencias en duración.

Por otra parte, una vez adquiridas las nociones simbólicas y lingüísticas ha sido objeto de debate la forma en que se desarrollan los conceptos de tiempo, así como la evolución del conocimiento sobre la perspectiva temporal, implícita muchas veces en el lenguaje, y las capacidades para medir el tiempo y para representar la estructura temporal de los sucesos y conocer el tiempo convencional.

Mientras que los defensores de la teoría piagetiana (Piaget, 1946) se centran en las carencias de quien resuelve el problema temporal sin tener en cuenta las demandas de la tarea, los teóricos de los factores interfirientes (Levin, 1989; Levin, Girat y Darom, 1980; Levin y Wilkening, 1989) insisten más en las demandas propias de la tarea de juicio temporal, sin tener en cuenta las características de la persona; la aproximación más equilibrada de las relaciones conceptuales (Richards, 1982) recoge la interacción entre esos dos aspectos e insiste en el cambio evolutivo que se produce en el conocimiento tanto de las relaciones entre el concepto de tiempo y las demás dimensiones, como en el de las propiedades 
únicas del mismo. Además, se ha comprobado que los niños pequeños tienen un sentido directo, no inferencial, de la duración (Richie y Bickhard, 1988) y que la forma de representar temporalmente los sucesos y de pensar sobre el pasado y el futuro cambia con la edad.

Durante la adolescencia la conciencia del tiempo aumenta. Las personas nos hacemos más reflexivas; sopesamos más el pasado y anticipamos y planificamos más el futuro, siendo además claramente conscientes de las interrelaciones y de la continuidad entre el pasado, el presente y el futuro. Las variables contextuales relacionadas con condicionantes socioculturales adquieren un gran peso sobre la naturaleza de las perspectivas temporales que cada adolescente mantiene. Al final de la adolescencia, las características y complejidades de la noción de tiempo conseguida son básicamente las mismas que las que presenta la cognición temporal de los adultos.

Como se ha podido constatar a lo largo de esta revisión, las personas tenemos una conciencia temporal que evoluciona paulatinamente a lo largo del desarrollo. Al igual que sucede con el resto de las propiedades de la información, el ser humano, en su continua adaptación al entorno, progresa en el modo en que se relaciona y comprende la temporalidad hasta llegar a la sofisticación del conocimiento temporal puesto de manifiesto en los adultos. El ser humano aprende a manejarse y a manejar el tiempo y ese dominio progresivo le ayuda a conocer la realidad. Ese papel tan fundamental del tiempo en cuanto que dominio de experiencia constituye un reclamo para dedicarle más investigación en el dominio psicológico.

Concluiremos diciendo que una gran parte de la bibliografía sobre el desarrollo de los aspectos temporales puede conceptualizarse como exploratoria puesto que como es un área que no se ha estudiado demasiado, los investigadores se han visto obligados a adoptar un acercamiento descriptivo para comprender las características básicas del tiempo. Una vez que se conocen los cambios y continuidades evolutivos principales, resulta posible empezar a estudiar la naturaleza de los procesos subyacentes. Sólo conociendo las características y los procesos que operan se podrá, finalmente, explicar el patrón evolutivo. Precisamante, es en el paso desde el trabajo descriptivo al explicativo donde se encuentra la investigación sobre la mayor parte de los conceptos temporales y donde habrá que centrar los esfuerzos en un futuro inmediato. De cualquier modo, el análisis evolutivo, ya proceda de la investigación exploratoria o de los estudios guiados por teorías, constituye una fuente de información fundamental sobre la naturaleza del conocimiento temporal.

\section{Referencias}

Acredolo, C. y Schmid, J. (1981). The understanding of relative speeds, distances, and durations of movement. Developmental Psychology, 17, $490-493$.

Anderson, N. H. (1981). Foundations of information integration theory. New York: Academic Press.

Antinucci, F. y Milier, R. (1976). How children talk about what happened. Joumal of Child Language, 3, 167-189.

Bauer, P. J. y Mandler, J. M. (1989). One thing follows another: Effects of temporal structure on 1- to 2-year-olds' recall of events. Developmental Psychology, 25, 197-206.

Block. R. A. (1978). Remembered duration: Effects of event and sequence complexity. Memory E Cognition, 6, 320-326.

Bloom, L., Lifter, K. y Hafitz. J. (1980). Semantics of verbs and the development of verb inflection in child language. Language, 56, 386-412. 
Bortner, R. W. y Hutrsch, D. F. (1972). Personal time perspective in adulthood. Developmental Psychology, 7, 98-103.

BrackBILL, Y. y FitzGerald, H. E. (1972). Stereotype temporal conditioning in infants. Psychophysiology, 9, 659-577.

Brackbill, Y., Fitzgerald, H. E. y Lintz, L. M. A. (1967). A developmental study of classical conditioning. Monographs of the Society for Research in Child Development, 32, $\mathrm{n}^{\circ} 8$.

Brackbill, Y., Lintz, L. y Fitzgerald, H. E. (1968). Differences in the autonomic and somatic conditioning of infants. Psychosomatic Medicine, 30, 193-201.

Bronckart, J. P. y Sinclair, H. (1973). Time, tense, aspect. Cognition, 2, 107-130

BuENo, M. B. (1990). El esfuerzo de procesamiento y los cambios en el contexto cognitivo en la estimación de la duración psicológica. Tesis de doctorado inédita. Salamanca: Universidad de Salamanca.

BüHLER, C. (1968). The course of human life as a psychological problem. Human Development, 11, 184-200.

BunL, W. E. (1971). Time, tense, and the verb. Berkeley: Universidad de California Press.

Bullock, M., Gelman, R. y Ballarargeon, R. (1982). The development of causal reasoning. En W. J. Friedman (Ed.), The developmental psychology of time (pp. 209-254). Nueva York: Academic Press.

Carranza, J. A., Brito de la Nuez, A. G. y Torrechlla, G. (1984). Un estudio sobre la comprensión de términos espacio-temporales en niños de 4 a 6 años. Infancia y Aprendizaje, 26, 111-119.

Case, R., SANDIESON, R. y DenNis, S. (1986). Two cognitive-developmental approaches to the design model of verification. Psychological Review, 82, 45-73.

Clark, E. (1971). On the adquisition of the meaning of before and after. Journal of Verbal Learning and Verbal Behavior, 10, 266-275.

Condon, W. y SANDER, L. (1974a). Neonate movement is synchronized with adult speech: Interactional participation in language acquisition. Science, 183, 99-101.

CONDON, W. y SANDER, L. (1974b). Synchrony demonstrated between movements of the neonate and adult speech. Child Development, 45, 456-462.

Comte, T., Howard, P. y Pleck, J. (1969). Adolescent perception of time: The effect of age, sex, and social class. Joumal of Personality, 37, 636-650.

CREPAULT, J. (1978a). Le raisonnement cinématique chez le préadolescent et l'adolescent. I. Esquisse d'un modèle théorique: concepts de base. Archives de Psychologie, 178, 133-183.

CREPAUtr. J. (1978b). Le raisonnement cinématique chez le préadolescent et l'adolescent. II. Prédictions du modèle théorique pour une classe de situations. Archives de Psychologie, 179, 185-203.

CREPAULT,J. (1979). Influence du repérage sur la durée; Etude génétique des inférences cinématiques. L' Année Psychologique, 79, 43-64.

CREPAULT.J. (1983). Modèles, raisonnements et notion de temps chez l'adolescent (Colloque sur la psychogenèse du temps, Genève). Cabiers de Psychologie cognitive, 3, 387-392 y 394-396.

Cromer, R. (1971). The development of the ability to decenter in time. British Joumal of Psycho$\log y, 62,353-365$.

Crowder, A. M. y Hohle, R. H. (1970). Time estimation by young children with and without informational feedback. Journal of Experimental Cbild Psychology, 10, 353-365.

DaviES, F. C. (1981). Ontogeny of circadian rhythms. En: J. Aschoff (Ed.), Handbook of behavioral neurobiology, vol. 4, (pp. 257-274). Nueva York: Plenum Press.

Day,J., Stein, N. L. y Trabasso. T. (1979). A study of inferential comprehension: The use of a story schema to remember pictures. Paper presentado en las reuniones de la Sociedad para la Investigación sobre el Desarrollo Infantil. San Francisco.

Demany, L., McKenZie, B. y VurpiLlot, E. (1977). Rhythm perception in early infancy. Nature, 266, 718-719.

DowD, J. M. у TRONIK, E. Z. (1986). Temporal coordination of arm movements in early infancy: Do infants move in synchrony with adult speech? Child Development, 57, 762-776.

Estaun-Ferrer, S. (1982). La compéhension des indices temporels chez l'infant. Tesis de doctorado inédita. París: Universidad René Descartes.

Estaun-Ferrer, S. (1985). Hacia una cronopsicología. Cuadernos de Psicología, 9, 201-207.

Estaun-Ferrer, S. (Octubre, 1991). The understanding of temporal relationships. Póster presentado en el encuentro sobre Tiempo, Acción y Cognición. Francia, Saint-Malo.

Fitzgerald, H. E., Lintz, L. M., Brackbill, Y. y Adams, G. (1967). Time perception and conditioning an autonomic response in human infants. Perceptual and Motor Skills, 24, 479-486.

FraISSE, P. (1948). Etude comparée de la perception et de l'estimation de la durée chez les enfants et les adultes. Enfance, 1, 199-211.

FraIsSE, P. (1957). Psychologie du temps. París: Presses Universitaires de France (Traducción inglesa: The psychology of time). Nueva York: Harper \& Row, 1963.

Frasse, P. (1982). The adaptation of the child to time. En: W. J. Friedman (Ed.), The developmental psychology of time (pp.113-139). New York: Academic Press.

FraIsSE, P. y OrsinI, F. (1958). Etude expérimentale des conduites temporelles. III: Etude génétique de l'estimation de la durée. L'Année Psychologique, 58, 1-6.

FrIEDMAN, W. J. (1977). Judgments of time intervals by young children. Perceptual and Motor Skills, $45,715 \cdot 720$.

Friedman, W. J. (1978). Development of time concepts in children. En H. W. Reese y L. P. Lipsitt (Eds.), Advances in child development and behavior (Vol. 12). Nueva York: Academic Press. 
Friedman. W. J. (1982). Conventional time concepts and children's structuring of time. En W. J. Friedman (Ed.), The developmental psychology of time (pp. 171-208). Nueva York: Academic Press.

Friedman. W. J. (1986). The development of children's knowledge of temporal structure. Child Development, $57,1386-1400$.

FRIEDMAN. W. J. (1989). The representation of temporal structure in children, adolescents and adults. En I. Levin y D. Zakay (Eds.), Time and Human Cognition. A Life-Span Perspective (pp. 259-304). North-Holland: Elsevier Science Publishers B.V.

Friedman, W. J. (1991). The development of children's representations of temporal structure. Paper presentado en el encuentro sobre Tiempo, Acción y Cognición. Francia, Saint-Malo.

Gardner, J. M. y Karmel, B. Z. (1984). Arousal effects on visual preferences in neonates. Deve. lopmental Psychology, 20, 374-377.

Goldstone, S. y Goldfar, J. L. (1966). The perception of time by children. En: A. H. Kidd y J. L. Rivoire (Eds.), Perceptual development in children (pp. 445-487). Nueva York: International Universities Press.

GreEne, A. L. (1986). Future-time perspective in adolescence: The present of things future revisited. Joumal of Youth and Adolescence, 15, 99-113.

Harner, L. (1975). Yesterday and tomornow: Development of early understanding of the terms. Developmental Psychology, 11, 864-865.

HARNER, L. (1980). Comprehension of past and future reference revisited. Journal of Experimental Child Psychology, 29, 170-182.

HARNER, L. (1982). Talking about the past and the future. En W. J. Friedman (Ed.), The developmental psychology of time (pp. 141-169). Nueva York: Academic Press.

Hicks, R. E., Miller, G. W. y Kinsbourne. M. (1976). Prospective and retrospective judgments of time as a function of amount of information processed. American Joumal of Psychology, 89, $719-730$.

JASNOw, M. y FeldSTEIn, S. (1986). Adult-like temporal characteristics of mother-infant vocal interactions. Child Development, 57, 754-761.

Kaufmann, F., Stucki, M. y Kaufmann-Hayoz, R. (1985). Development of infants' sensitivity for slow and rapid motions. Infants Bebavior and Development, 8, 89-98.

Lester, B. M., Hoffman, J. y Brazelton, T. B. (1985). The thythmic structure of mother-infant interaction in term and preterm infants. Child Development, 56, 15-27.

Levin, I. (1977). The development of time concepts in young children: Reasoning about duration. Child Development, 48, 435-444.

LEVIN, I. (1979). Interference of time-related and unrelated cues with duration comparisons of young children: Analysis of Piaget's formulation of the relation of time and speed. Child Development, 50, 469-477.

LEVIN, I. (1982). The nature and development of time concepts in children: The effects of interfering cues. En W. J. Friedman (Ed.), The developmental psychology of time (pp. 47-85). Nueva York: Academic Press.

Levin. I. (1989). Principles underlying time measurement: The development of children's constraints on counting time. En I. Levin y D. Zakay (Eds.), Time and buman cognition: A life-span perpective (pp. 145-183). North-Holland: Elsevier Science Publishers B.V.

Levin, I. y WILKENING, F. (1989). Measurement time via counting: the development of children's conceptions of time as a quantifiable dimension. En I. Levin y D. Zakay (Eds.), Time and buman cognition: A life-span perspective (pp. 119-144). North-Holland: Elsevier Science Publishers B.V.

Levin, I., Gilat, I. y Darom, E. (1980). The role of cue salience in the development of time concepts: Duration comparisons in young children. Developmental Psychology, 16, 661-671.

Levin, I., Israeli, E. y Darom, E. (1978). The development of time concepts in young children: The relation between duration and succession. Child Development, 49, 755-764

Levin, I., W WLKening, F. y Dembo, Y. (1984). Development of time quantification: Integration and non-integration of beginnings and endings in comparing durations. Child Development, 55 , 2160-2172.

LEw/KOWICZ, D. J. (1985a). Developmental changes in infants' response to temporal frequency. $D e$ velopmental Psychology, 21, 858-865.

Lewkowicz, D. J. (1985b). Bisensory response to temporal frequency in 4 -month-old infants. Developmental Psychology, 21, 306-317.

LEwrowicz, D. J. (1986). Developmental changes in infants' bisensory response to synchronous durations. Infant Behavior and Development, 9, 335-353.

LewKowicz, D. J. (1988a). Sensory dominance in infants: 1 . Six-month-old infants' response to auditory-visual compounds. Developmental Psychology, 24, 155.171.

Lewkowicz. D. J. (1988b). Sensory dominance in infants: 2. Ten-month-old infants' response to auditory-visual compounds. Developmental Psychology, 24, 172-182.

LEWKowicz, D. J. (1989). The role of temporal factors in infant behavior and development. En I. Levin y D. Zakay (Eds.), Time and human cognition: A life-span perspective (pp. 9-62). NorthHolland: Elsevier Science Publishers B.V.

Lovell, K. y Stater, N. (1960). The growth of the concept of time: A comparative study. Child Psycbology and Psyquiatry, 1, 179-190.

MAnder, J. M. y Johnson, N. S. (1977). Rememberance of things parsed: Story structure and recall. Cognitive Psychology, 9, 111-151. 
Marquis, D. P. (1941). Learning in the neonate. Journal of Experimental Psychology, 29, 263-282.

Mendelson, M. J. (1986). Perception of the temporal pattern of motion in infancy. Infant Behavior and Development, 9, 231-243.

Miliar, W. S. y W WTSON, J. S. (1979). The effect of delayed feedback on infant learning reexamined. Child Development, 50, 747-751.

Miller, G. A. (1978). Pastness. En G. A. Miller y E. Lennenberg (Eds.), Psychology and Biology of Language and Thought (pp. 167-185). Nueva York: Academic Press.

Moerk, E. (1974). Age and epogenic influences on aspirations of minority and majority group children. Joumal of Counseling Psychology, 21, 294-298.

Moerk. E. y BeCKer, P. (1971). Attitudes of high school students toward future marriage and college education. The Family Coordinator, 20, 67-73.

Montangero, J. (1984). Perspectives actuelles sur la psychogenèse du temps. L'Année Psychologique, 84, 433-460.

Morrongiello, B. A. (1984). Auditory temporal pattern perception in 6-and 12-month-old infants. Developmental Psychology, 20, 441-448.

Nurmi, J. E. (1989). Planning, motivation, and evaluation in orientation to the future: A latent structure analysis. Scandinavian Joumal of Psychology, 30, 64-71.

Ornstein, R. E. (1969). On the experience of time. Harmondsworth: Penguin.

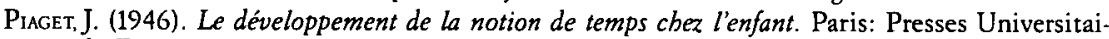
res de France.

PIAGET, J. (1954). The construction of reality in the child. Nueva York: Basic Books.

PIAGET, J. (1960). The language and thought of the child. Londres: Routledge y Kegan Paul. (Publicado originalmente en 1923).

PIAGET, J. (1969). The child's conception of time. Londres: Routledge y Kegan Paul.

PiAget, J. (1979). Introducción a la epistemología genética. 2. El pensamiento físico. Buenos Aires: Paidós.

Pouthas, V. (1981). Adaptation à la durée chez l'enfant de 2 à 5 ans. L'Année Psycbologique, 81, 33-50.

Pouthas, V. (1985). Timing bebavior in young cbildren: A developmental approach to conditioned spaced responding. En: J. A. Michon y J. L. Jackson (Eds.), Time, Mind, and Behavior (pp. 100-109). Heidelberg: Springer-Verlag.

Ramey, C. T. y OURTh, L. L. (1971). Delayed reinforcement of vocalization rates in infants. Child Development, 42, 291-297.

Richands, D. D. (1982). Children's time concepts: Going the distance. En: W. J. Friedman (Ed.), The developmental psychology of time (pp. 13-45). Nueva York: Academic Press.

Richie. D. M. y Bickhard, M. H. (1988). The ability to perceive duration: Its relation to the development of the logical concept of time. Developmental Psychology, 24, 318-323.

SACHS, J. (1979). Topic selection in parent-child discourse. Discourse Processes, 2, 145-153.

Seniger, R. (1988). Adolescents' orientation toward the future: Sex role differentiation in a sociocultural context. Sex Roles, 18, 739-757.

SETH, U. (1986). Future time orientation of boys and girls of different educational faculties. In dian Joumal of Cument Psychological Research, 1, 107-111.

SiEgler, R. S. y McGilly, K. (1989). Strategy choices in children's time-telling. En I. Levin y D. Zakay (Eds.), Time and human cognition. A life-span perspective (pp. 185-218). North-Holland: Elsevier Science Publishers B.V.

Siegler, R. S. y Richards, D. D. (1979). Development of time, speed, and distance concepts. Developmental Psychology, 15, 288-296.

$\mathrm{S}_{\text {MITH, }}$ C. (1980). The acquisition of time talk: Relations between child and adult grammars. Joumal of Child Language, 7, 263-278.

Stamps, L. E. (1977). Temporal conditioning of heart rate responses in newborn infants. Developmental Psychology, 13, 624-629.

Stamps, L. E. y Porges, S. W. (1975). Heart rate conditioning in newborn infants: Relationships among conditionability, heart rate variability, and sex. Developmental Psychology, 11, 424.431.

Stein, N. L. y Glenn, C. G. (1979). An analysis of story comprehension in elementary school chil. dren. En: R. O. Freedle (Ed.), New directions in discourse processing (Vol. 2). Norwood, N. J. : Ablex.

Stein, N. L. y Glenn, C. G. (1982). Children's concept of time: The development of a story schema. En: W. J. Friedman (Ed.), The developmental psychology of time (pp. 255-282). Nueva York: Academic Press.

Stein, N. L. y Trabasso, T. (1981). What's in a story: Critical issues in story comprehension. En R. Glaser (Ed.), Advances in the psychology of instruction. Hillsdale, N. J. : Erlbaum.

Stern, C. y Stern, W. (1907). Die kindersprache. Leipzig: Barth.

Stern, D. N. y GibBon, J. (1979). Temporal expectations of social behavior in mother-infant play. En E. B. Thoman (Ed.), Origins of the infant's social responsiveness. Hillsdale, N. J.: Erlbaum.

Stern, D. N., Beebe, B., Jaffe, J. y Bennett, S. L. (1977). The infant's stimulus world during social interaction: A study of caregiver behaviors with particular reference to repetition and timing. En H. R. Shaffer (Ed.), Studies in mother-infant interaction. Nueva York: Academic Press.

Sundberg, N. D., Poole, M. E. y Truer, L. E. (1983). Adolescents' expectations of future events: 
A cross-cultural study of Australians, Americans, and Indians. Intemational Joumal of Psycho$\log y, 18,415.427$.

Thelen, E. (1979). Rhythmical stereotypies in normal human infants. Animal Bebavior, 27, 699-715.

Thelen, E. (1981). Rhythmical behavior in infancy: An ethological perspective. Developmental Psychology, 17, 237-257.

Turco. T. L. y Stamps. L. E. (1980). Heart rate conditioning in young infants using a visual conditional stimulus. Joumal of Experimental Child Psychology, 29, 117-125.

Vasquez, A. (1982). Temps social/ Temps cultural. Enfance, 5, 335-350.

WASHBUR, K. J. y COHEv, L. B. (1984). Infant perception of rhythmic form. Artículo presentado en la International Conference on Infant Studies, Nueva York.

Wrason. J. S. (1979). Perception of contingency as a determinant of social responsiveness. En E. Thoman (Ed.). The origins of social responsiveness (pp. 33-64). Hillsdale, N. J. : Lawrence Erlbaum Associates.

W Atson, J. S. (1984). Bases of causal inference in infancy: Time, space, and sensory relations. En L. P. Lipsitt y C. Rovee-Collier (Eds.), Advances in infancy research, Vol. 13, (pp. 152-165). Norwood, N. J.: Ablex Publishing Corp.

WEIST, R. M. (1989). Time concepts in language and thought: Filling the piagetian void from two to five years. En I. Levin y D. Zakay (Eds.), Time and buman cognition. A life-span perspective (pp. 63-118). North-Holland: Elsevier Science Publishers B.V.

W ILKENING, F. (1979). Combining of stimulus dimensions in children's and adult's judgments of area: An information integration analysis. Developmental Psychology, 15, 25-33.

W ILKENING, F. (1981). Integrating velocity, time, and distance information: A developmental study. Cognitive Psychology, 13, 231-247.

Wilkening. F, Levin, I. y Druyan, S. (1987). Children's counting strategies for time quantification and integration. Developmental Psychology, 23, 823-831.

\section{Extended summary}

The study of the development of subjective time plays an important role in any general consideration of perceptual and cognitive development, for time is an ever present property of sensory experience.

Although for many years human knowledge of temporality was thought to demand a mastery of symbolic and linguistic notions, recent research on this topic indicates that infants do have temporal abilities that enable them to develop a series of affective, perceptual, cognitive, linguistic, and social behaviours in order to adapt well to the environment. Six month-old infants frequently exhibit stereotypical movements that point to their early sensitivity to rhythm and which serve as a means of communication with their caregivers. At only a few months of age infants also form temporal expectancies concerning communicative exchanges with their caregivers. It is even possible that in very young infants, the movements of different parts of their body are synchronized with the adult's speech. In the prelinguistic period, children's ability to order events in a temporal sequence has already emerged. A little later, the first notions of a temporal perspective are acquired. Additionally, infants possess basic discriminative abilities related to their perception of time, including an ability to detect differences in duration.

In the numerous studies on the development of a time knowledge after symbolic and linguistic notions have been acquired, there are several foci of interest, among them: controvertial theories on a «time concept»; evolution of a temporal perspective; development of the capacity to measure time; mode of representation of a temporal structure of events; and knowledge of conventional time. Piagetian theoreticians (Piaget, 1946, 1969) focus on the problem solver's deficits without taking into account the demands made by the task. Then 
1989) focus instead on the demands made by the time judgement task. The interaction between these two views is reflected on a more balanced approach, namely, a conceptual approach (Richards, 1982). This approach stresses the developmental change produced through learning the unique aspects of the concept itself and not just through the relationships betwen the time concept and related concepts. In addition, children have been observed to hava a direct, noninferential, sense of duration. The mode of temporal representation of events, and the mode of thinking about about past and future events also change with age.

During adolescence, there is an increase in time awareness. As a result more planning for future events takes place than during the earlier childhood years. Contextual variables related to sociocultural conditions markedly influence the nature of adolescents' temporal perspectives. Once adolescence ends, the characteristics and complexities of the time notion acquired are basically the same as those present in adult temporal cognition.

From the work carried out up to the present, we know how children perform at different ages. However, underlying processes leading to change are still not well known. Thus, it is necessary to carry out studies that attempt to explain the developmental pattern of temporal knowledge rather than undertake merely descriptive ones. 